\section{GW23-e0675 RELATIONSHIP OF ARTERIAL STIFFNESS AND LEFT VENTRICULAR DIASTOLIC DYSFUNCTION IN CORONARY HEART DISEASE PATIENTS WITHOUT MYOCARDIAL INFARCTION}

doi:10.1136/heartjnl-2012-302920o.20

Mu Lin, Yongquan Wu. BeiJing Friendship Hospital, Capital Medical University, Cardiovascular Center

Objectives The carotid-femoralpulse wave velocity (cfPWV) has been regarded as a marker of arterialstiffness. The present study was conducted to evluate the association betweencfPWV and left ventricular dialstolic dysfunction and its severity in coronaryheart disease patients without myocardial infarction.

Methods From August 2010 until August 2011, we enrolled 243 patients who are hospitalised for performing coronary angiography. Among these in patients, who with ejection fraction $\leq 50 \%$, heart failure, organic heart diseases, atrialfibrillation, acute myocardial infarction, peripheral arterial diseases, chronic obstructive pulmonary disease, renal insufficiency (serum creatinine $>1.5 \mathrm{mg} / \mathrm{dl}$ ) were excluded, the other 172 subjects were included. Allpatients' medical history and general clinical data were recorded. Bloodsampling was performed in the morning after a $12 \mathrm{~h}$ overnight fast. In these patients, we sequentially measured echocardiography, cfPWV, carotid intima media thickness and coronary angiography. Left ventricular diastolic dysfunction was divided into four groups according to the results of echocardiography:38 subjects with normal (Non-HFNEF), 45 subjects with mild dysfunction (Grade I), 57 subjects with moderate dysfunction (Grade II) and 32 subjects with severe dysfunction grade (Grade III). Based on the number of stenotic vessels on coronaryangiography, these patients were divided into three groups: 1-vessel, 2-vessel and 3 -vessel groups. Data was analysed using SPSS 11.5 software.

\section{Results}

1. The patients in diastolic dysfunction groupwere much older and had significantly higher prevalence of hypertension, diabetes mellitus, hyperlipemia and smoking together with higher body mass index compared with those in normal diastolic function group $(\mathrm{p}<0.001)$;

2. There were significantdifferences in theseverity of coronary heart disease between four groups, the number of affected coronary arteries wascorrelated with the severity of diastolic dysfunction $(\mathrm{p}<0.001)$;
3. The transmission speed of carotid - femoral artery pulse wave, the film thicknessof the carotid artery, the inner diameter of left atrium, the thickness ofatrial septal basal segment and left ventricular wall as well as the leftentricular mass index were significantly increased with the exacerbation ofdiastolic dysfunction between four groups $(p<0.001)$. There were alsosignificant differences in the ultrasonography index such as E/A, E/E1 and Teiindex that could indirectly reflect left ventricular diastolic function betweenfour groups $(\mathrm{p}<0.05)$;

4. There weresignificant differences in the transmission speed of carotid - femoral arterypulse wave between different groups with coronary heart disease $(7.84 \pm 0.46$ vs $10.25 \pm 1.34$ vs $14.46 \pm 1.68$, $\mathrm{p}<0.001$ );

5. cfPWV was positively correlated withE/E' $(\mathrm{r}=0.851, \mathrm{p}<0.001)$, the film thickness of the carotid artery $(r=0.581, p<0.001)$, theinner diameter of left atrium $(r=0.349, p<0.01)$ and the leftentricular mass index $(r=0.256, p<0.01)$.

6. The area under ROC of thetransmission speed of carotid - femoral artery pulse wave to reflect leftventricular diastolic dysfunction was 0.908 (95\% CI 0.995 to 1.000$)$. If $8.65 \mathrm{~m} / \mathrm{s}$ was selected as the cutoff value, the sensitivity and specificityto diagnose left ventricular diastolic dysfunction was $93.8 \%$ and $97.2 \%$, respectively.

\section{Conclusions}

1. Oldage, high blood pressure, diabetes, hyperlipidaemia, smoking and overweight werepossible risk factors of diastolic dysfunction in patients with coronary arterydisease.

2. The leftventricular diastolic functionwas declined following the increased severity of affected coronary arteries inpatients with coronary heart disease.

3. The arterialstiffness wasincreased in patients with coronary heart disease, and correlated with the severity of coronary lesions.

4. The arterialstiffness wascorrelated with theseverity of carotid atherosclerosis and left ventricular diastolic function in patients with coronary heartdisease, which could independently reflect the severity of atherosclerosis andleft ventricular diastolic dysfunction. 